depression is powerless to draw it towards the centre. Only when its velocity is decreased by friction does the barometric gradient, just before produced by the centrifugal force, urge the wind inwards to the middle of the storm. The additional gradient, therefore, represents potential energy, derived from the actual energy of the rotating winds, and all ready to be transformed into actual energy again, as soon as friction has destroyed some of the velocity of rotation.

The general interaction of the storm-forces may now be thus summarized: in obedience to a centripetal tendency, produced by differences of temperature or of pressure, or both, the air moves along the surface to the region of low pressure. On its way, the deflective force arising from the earth's rotation turns it continually to one side, and so gives it a more and more nearly circular path ; and, in addition to this, its rotary velocity increases as much as its radius of rotation decreases: the tangential component of its spiral motion must therefore continually increase. With the increase of this component, and the decrease of the radius of rotation, the centrifugal force $\left(v^{2} \div v\right)$ must increase rapidly, and soon come to equal and counterbalance the original centripetal force, and at the same time greatly increase the barometric gradients. At this point the wind would blow in a circular path, were it not that friction.with the sea or ground is continually consuming some of its velocity, and thus decreasing its centrifugal force, and allowing the potential energy of the steep barometric gradient to produce centripetal motion. This decreases its radius, and at once gives it new life, again to be partly destroyed and renewed as before. Absolutely circular motion can therefore never be attained, although it is approached very closely near the centre. At sea, where friction is small, and in tropical latitudes, where the strength of the storm is great, the wind is unable to reach the storm-centre; for, when the distance from the centre is reduced to only five or ten miles, the centrifugal force is so great, and the wind's course is so nearly circular, that it is carried aloft by the up-draught before it can enter noticeably farther: the central area is therefore left unprovided with violent winds, and is generally a comparative calm, known as the 'eye of the storm,' of which there will be more to say later. The general form of the storm-wind's spiral can be deduced from the preceding considerations. The angle between the tangential component and the actual path of the wind, which is called the inclination (fig. 11.), will vary with the relation of the circular and centripetal elements of the wind's motion ; the tangent of the inclination will equal the radial divided by the tangential component: hence in the outer part of the storm the inclination will be large, and the wind will blow almost directly toward the storm-centre; but nearer the centre the inclination will become smaller and smaller, and the wind will blow in a more and more nearly circular path. It will also be understood, that the upper winds, less influenced by friction, will near the centre have a greater velocity and a less inclination than the lower ones. Moreover, the inward gradient which they produce will be effective and important in urging along the slower surface-winds, in a manner better illustrated in a tornado, where this action will be more fully described.

(To be continued.)

\section{ON THE DEVELOPMENT OF TEETH IN THE LAMPREY.}

THE teeth in the myxinoid fishes are quite different from those of other vertebrates, and have hitherto been supposed to belong in an entirely different category. Nothing has been known with regard to their development, except a brief statement as to their mode of succession in Petromyzon by Professor Owen, in his 'Odontography.'

The teeth of the lamprey are horny, and of simple conical shape, disposed concentrically in the dome-shaped mouth. Besides these, there are horny lingual and palatal teeth.

The kindness of my friend Professor Benecke of Königsberg, who sent me a number of lampreys at the end of their metamorphosis from Ammocoetes, has enabled me to follow out the development of these horny teeth with unexpected results ; for, as far as the essential part of the process is concerned, it differs but slightly from the normal course of true dental development. There is first formed a low conical papilla of somewhat reticulate tissue, belonging to the mesoblast (m.p.), and continuous with the dermis, which in this, as in other vertebrates, is of mesoblastic origin. Over this papilla the epiblast which lines the cavity of the mouth becomes extremely thick, and consists of very numerous layers of cells. All of these layers can be continuously traced into the other epiblast of the mouth, as well as that of the external skin. In the stage here figured there may be seen, immediately overlying the mesoblastic papilla, a layer of epiblastic cells irregularly columnar and polygonal in shape (e.o.). These cells are the homologue of the 
enamel-organ of the other vertebrates, and originate in the same way. So far, at least, the lamprey does not show an essentially different type of tooth-development from that known in other groups.

The cells of the 'enamel-organ' are rapidly proliferating, and have thrown off from their outer surface a conical cap of cells $(2 d t$.$) ,$ which are flattened, and which show an incipient formation of pigment among them. This hollow cone of cells is the rudiment of the youngest tooth, which in the stage here described is the second of the series. Outside of the rudimentary tooth is a cone of polygonal epiblastic cells, several layers deep (i.e.); and this is again followed by the first tooth, now almost completely cornified and pigmented,

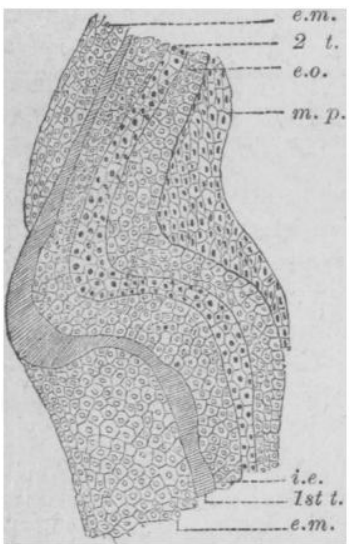

Section through inner side of $11 \mathrm{p}$ of metamorphosing lamprey. e.m., epiblast of mouth; 1st $t$. tooth; e.o., enamel-organ; i.e., intermediate epiblast-cells be tween successive teeth; m.p. mesoblastic papilla. oldest tooth; $2 d t$., youngest so that traces of cellular structure are but faintly discernible $(1 s t t$.$) . The$ tip of this tooth has just penetrated the skin of the mouth, and is elsewhere covered by the manylayered epiblast $(e$. $m$.).

We see, therefore, that the essential parts of the typical vertebrate tooth are here present; namely, the mesoblastic papilla, and the over-lying epiblastic enamel-organ. But the ordinary type of dental development is here greatly modi-

fied. The papilla is never ossified : and the enamel-organ secretes no enamel, but functions as a sort of tooth-gland, throwing off successive hollow cones of flattened and cornified epiblastic cells. The actual tooth of the lamprey is therefore not the homologue of the entire tooth of a selachian, but simply of the enamel-cap. It is not difficult, however, to understand how the process seen in Petromyzon could be derived from that in the selachian. In consequence of this change, another difference arises : as the papilla never ossifies or becomes protruded, it is no longer necessary that for every new tooth a new enamel-organ should be formed by budding from the old one ; so each enamel-organ is converted into a permanent tooth-gland, functional throughout the life of the animal.
This view of the peculiarities of dental development in Petromyzon implies, of course, that this group of fishes was derived from ancestors possessed of teeth of the ordinary or selachian type. Further, as it is now very generally adinitted that teeth are only modified placoid scales, it follows that the lampreys are descended, ultimately at least, from forms provided with placoid scales.

Such a conclusion, however, does not by any means commit us to the view that the myxinoids are degenerate descendants of some gnathostomatous group, as this is no more implied in the possession of ordinary calcareous teeth than in the presence of the horny teeth which the group has long been known to possess.

W. B. Scott.

Morphological laboratory, Princeton, N.J. Nov. $3,1883$.

\section{NORDENSKIÖLD ON THE INLAND ICE OF GREENLAND.1}

IN a series of letters to Mr. Oscar Dickson, Baron Nordenskiöld has given a detailed report of the leading incidents and results of his recent expedition, though it will still be some time ere we can learn what are the full gains to science. The leading novelty of the expedition was, of course, the journey into the interior of Greenland.

After landing Dr. Nathorst and his party at Waigatz Sound, Nordenskiöld went back to Egedesminde, which he reached on June 29. The following day he left for Auleitsivik Fjord, from which the expedition was to start. He then proceeds: -

On July 1 the Sophia anchored in the bay. We found here a splendid harbor with clay bottom, some seven fathoms deep, surrounded by gneiss rocks from six hundred to a thousand feet in height, the sides of which are in some places covered with low but close shrubs, or clothed with some species of willow, mosses, and lichen, which, when we arrived, were ornamented with a quantity of magnificent blossoms. From one of the slopes a torrent descended, the temperature of which was $12.3^{\circ} \mathrm{C}$. The weather was fine, the sky cloudless, and the air very dry. July 1 to 3 were employed in making preparations for the ice-journey, while the naturalists made excursions to various places in order to collect objects relating to the conditions of the country. On the night of the $3 d$ every thing was ready for a start; and, after some difficulty in reaching the spot where the baggage was, we were fairly off. The spot from which we set out on the journey was only five kilometres from the actual shore, and situaled below a little lake into which a number of glacier rivers fell. We proceeded up the river in a Berton boat, purchased in England. On the night of the 4th we camped for the first time on the ice. The expedition consisted of nine men besides myself. After a great deal of hard work in getting the sledges over the ice, which was here very 